\title{
Initial and Programmed Combination Therapy With Oral Drugs for Severe Idiopathic Pulmonary Arterial Hypertension
}

\author{
Hisataka MaKi, ${ }^{1}$ MD, Atsushi Yao, ${ }^{1} \mathrm{MD}$, Toshiro Inaba, ${ }^{1} \mathrm{MD}$, Taro ShIGA, ${ }^{1} \mathrm{MD}$, \\ Masaru Hatano, ${ }^{1}$ MD, Koichiro Kinugawa, ${ }^{1}$ MD, Takeshi Yamashita, ${ }^{2}$ MD, \\ Tadanori Aizawa, ${ }^{2} \mathrm{MD}$, and Ryozo NaGaI, ${ }^{1} \mathrm{MD}$
}

\begin{abstract}
SUMMARY
A 49-year-old woman suffering from rapidly progressing right-sided heart failure assessed as World Health Organization functional class (WHO-FC) IV is described. After treatment with oxygen and diuretics, she was in WHO-FC III on admission to our hospital, as confirmed by her poor exercise tolerance in cardiopulmonary exercise testing. Upon detailed examination, she was diagnosed as having idiopathic pulmonary arterial hypertension (IPAH). Right heart catheterization (RHC) revealed severe pulmonary hypertension ( $\mathrm{mPAP}=65 \mathrm{mmHg}$ ) with a markedly decreased cardiac index $\left(\mathrm{CI}=1.0 \mathrm{~L} / \mathrm{minute}^{2} \mathrm{~m}^{2}\right.$, and an acute vasoreactivity test with nitric oxide inhalation did not show any response. Due to her severe condition, we decided to attempt oral combination therapy consisting of bosentan, tadalafil, and beraprost, prescribed in the same order and titrated up to their maximum respective doses, instead of intravenous (IV) epoprostenol therapy. Her clinical symptoms improved day by day, and the hemodynamic parameters recovered to nearly normal ranges about 6 months after initiation of the combination therapy. Initial/programmed oral combination therapy for severe IPAH patients is not yet fully established, and there is less evidence concerning its efficacy than IV epoprostenol therapy. However, it has tremendous advantages for PAH patients when they respond well. It is very important to further identify what types of PAH patients will respond to this oral combination therapy and should be treated with it as the first-line therapy. (Int Heart J 2011; 52: 323-326)
\end{abstract}

Key words: Pulmonary arterial hypertension, Combination therapy, Bosentan, Tadalafil, Beraprost

$\mathrm{T}$ he molecular mechanism progressing pulmonary arterial hypertension (PAH) has been investigated widely in animal models and clinical cases, and abnormalities in 3 main pathways (prostacyclin - cyclic AMP, nitric oxide (NO) - cyclic GMP, and endothelin) are recognized as being important for the pathophysiology of PAH. Recently, drugs treating these 3 main pathways, such as prostacyclin analogues, endothelin receptor antagonists (ERA), and phosphodiesterase-5 inhibitors (PDE-5 inhibitor), have been approved for clinical use in PAH patients, and in fact have been shown to improve clinical symptoms, exercise tolerance, and hemodynamic status and prolong the time to clinical worsening in $\mathrm{PAH}$ patients. ${ }^{1)}$ Thus, the potential of combination therapy that treats all 3 main pathways seems to be more promising and should be considered for severe PAH patients. However, both the American College of Cardiology/American Heart Association $(\mathrm{ACC} / \mathrm{AHA})^{2)}$ and the European Society of Cardiology (ESC) $)^{3)}$ guidelines have recommended that IV epoprostenol should be used as the treatment of first choice for severe PAH patients with WHO functional class III and IV, while a PDE-5 inhibitor (sildenafil) or ERA (ambrisentan and bosentan) may be used as the first choice for WHO-FC III patients. However, sequen- tial combination therapy is commonly recommended as one of the second choices according to the response to the first drug. There may be questions why the initial/programmed combination therapy with an oral prostacyclin analogue, ERA, and PDE-5 inhibitor is not recommended before IV epoprostenol therapy, especially for severe PAH patients, because IV epoprostenol therapy involves risk for catheter/pump-induced troubles such as insufficient drug delivery and catheter infection as well as decreases the quality of life. The ESC guideline ${ }^{3)}$ does not provide detailed information concerning which drugs should be chosen as the initial combination therapy in WHOFC IV patients. It is therefore important to establish an effective combination therapy with oral drugs before adding IV epoprostenol to the treatment of PAH patients. Here we describe a severe PAH case who recovered dramatically in response to initial/programmed combination therapy with oral drugs treating the 3 major pathways.

\section{Case Report}

In December, 2009, a 49-year-old healthy woman com-

From the ${ }^{1}$ Department of Cardiovascular Medicine, Graduate School of Medicine, The University of Tokyo, and ${ }^{2}$ Department of Cardiology, Cardiovascular Institute, Tokyo, Japan.

This research is supported by the Japan Society for the Promotion of Science (JSPS) through its "Funding Program for World-Leading Innovative R\&D on Science and Technology (FIRST Program)".

Address for correspondence: Atsushi Yao, MD, Department of Cardiovascular Medicine, Graduate School of Medicine, The University of Tokyo, 7-3-1 Hongo, Bunkyo-ku, Tokyo 113-8655, Japan.

Received for publication January 26, 2011

Revised and accepted May 13, 2011. 


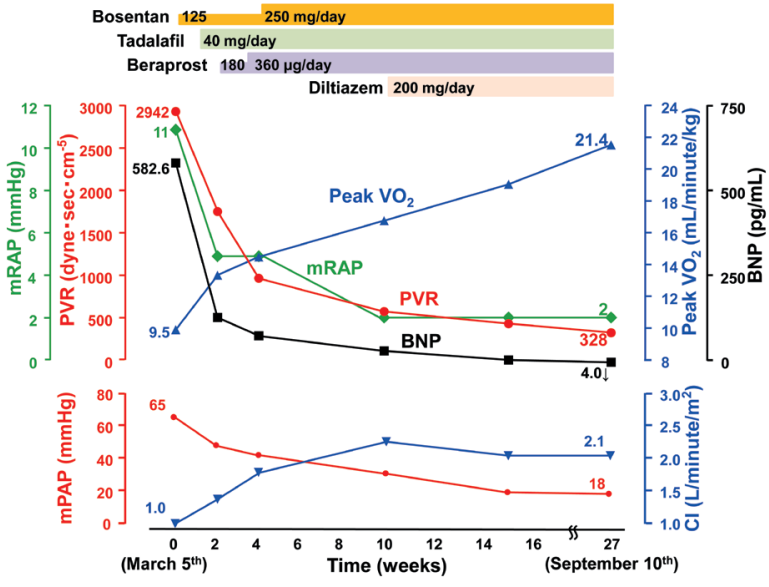

Figure 1. Time course of clinical parameters and drug administration. BNP indicates B-type natriuretic peptide; mRAP, mean right atrial pressure; PVR, pulmonary vascular resistance; $\mathrm{VO}_{2}$, volume of oxygen uptake; mPAP, mean pulmonary arterial pressure; and $\mathrm{CI}$, cardiac index.

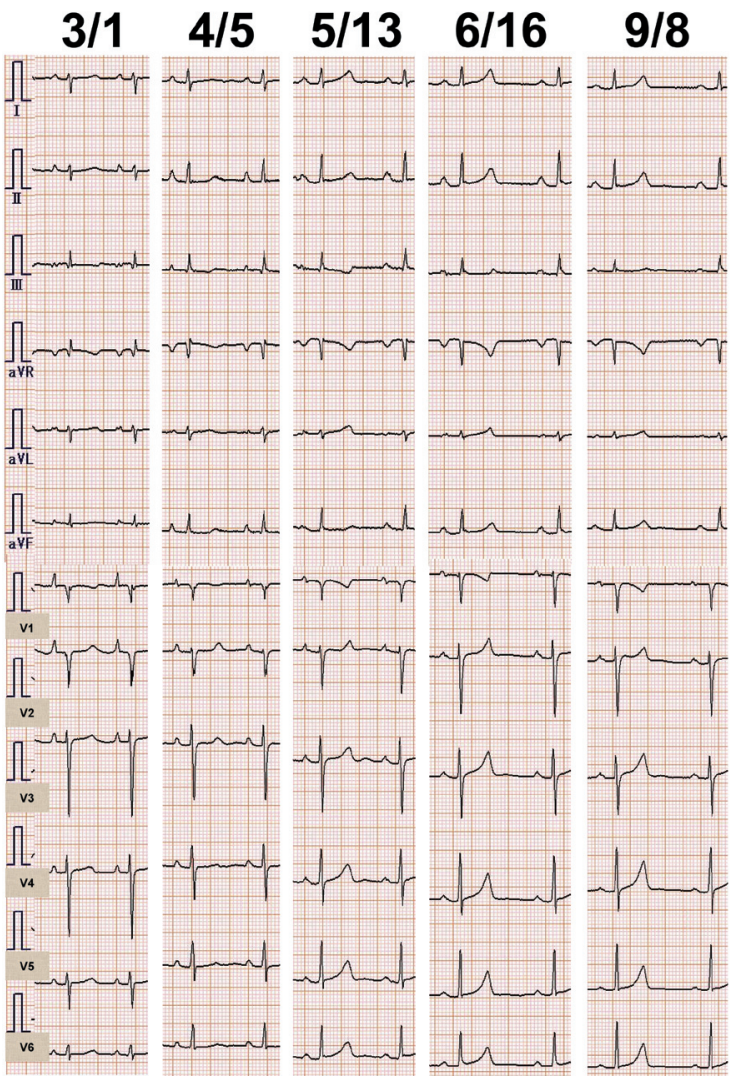

Figure 2. Change in electrocardiography (ECG) during the combination therapy. Before the combination therapy, the ECG showed right axis deviation and poor $\mathrm{R}$ wave progression with tall $\mathrm{P}$ waves in the precordial leads. These abnormal ECG findings improved over time and disappeared after 6 months of the combination therapy.

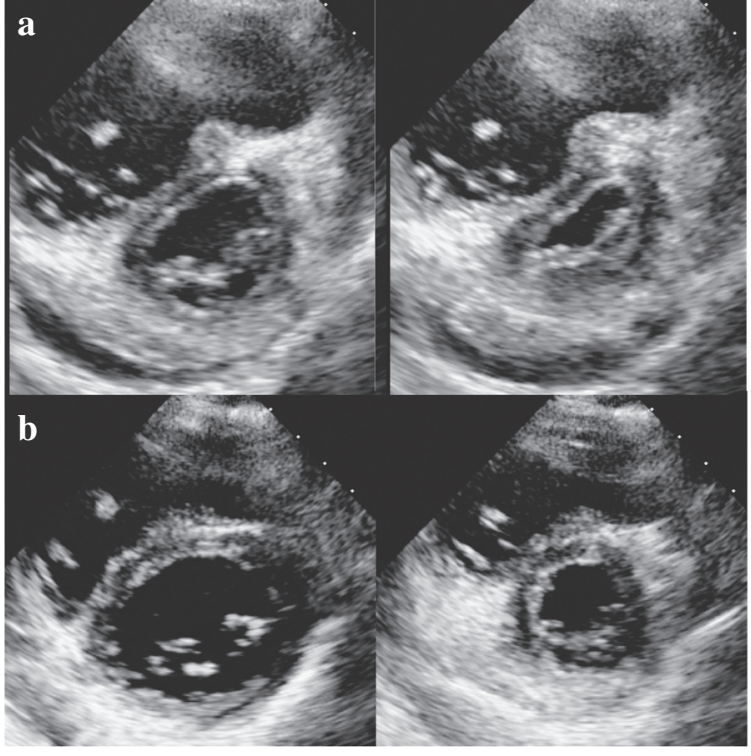

Figure 3. Features of echocardiogram before and after combination therapy. Before the combination therapy, echocardiography showed a markedly dilated right ventricle with a compressed left ventricle and pericardial effusion (a). These abnormal findings were almost normalized after 6 months of the combination therapy (b)

plained of exertional breathlessness, followed by the gradual emergence of bilateral leg edema. Since she could not walk more than 20 meters due to dyspnea [World Health Organization functional class (WHO-FC) IV], she visited a nearby hospital on February $24^{\text {th }}, 2010$. Her arterial oxygen saturation by pulse oxymetry was $92.6 \%$ in room air and the plasma level of B-type natriuretic peptide (BNP) was $1604 \mathrm{pg} \cdot \mathrm{mL}^{-1}$. She was treated with oxygen and diuretics after hospitalization. Electrocardiography showed rightward QRS axis orientation and prominent $\mathrm{P}$ waves in $\mathrm{V}_{1}$-lead. Transthoracic echocardiography demonstrated dilatation of the right atrium and right ventricle with a compressed left ventricle, and elevation of the tricuspid regurgitation pressure gradient $(90 \mathrm{mmHg})$. The results of other medical examinations did not indicate there was a specific cause leading to pulmonary hypertension $(\mathrm{PH})$. Based on these findings, she was suspected of having idiopathic PAH (IPAH) and transferred to our hospital on March $1^{\text {st }}$. Her clinical course is summarized in Figure 1. She was in WHO-FC III, also confirmed by a severely decreased peak $\mathrm{VO}_{2}(9.5 \mathrm{~mL} /$ minute/ $/ \mathrm{kg})$. The first hemodynamic study (March $5^{\mathrm{th}}$ ) showed severe $\mathrm{PH}$ (mean pulmonary arterial hypertension: $\mathrm{mPAP}=65 \mathrm{mmHg}$ ) with a normal pulmonary wedge pressure, and a markedly reduced cardiac index $\left(\mathrm{CI}=1.0 \mathrm{~L} \cdot \mathrm{min}^{-1} \cdot \mathrm{m}^{-2}\right)$, resulting in highly elevated pulmonary vascular resistance $\left(\mathrm{PVR}=2942\right.$ dyne $\left.\cdot \mathrm{sec} \cdot \mathrm{cm}^{-5}\right)$. An acute vasoreactivity test was negative with no response to inhaled nitric oxide (NO). Therefore, we diagnosed her as having severe IPAH, and decided to attempt a combination therapy consisting of 3 types of oral drugs as the first-line therapy, rather than IV epoprostenol. We began with bosentan administration, and then added tadalafil and beraprost sequentially over a two week period, followed by up-titration of beraprost and bosentan to their maximum dosages two weeks later. Her clinical symptoms were relieved, and the fourth hemodynamic 
study (May $14^{\text {th }}$ ) revealed a marked improvement in PVR and almost normalized CI. Moreover, an acute vasoreactivity test, for the first time, showed an apparent response (19\% reduction in PVR), so we added diltiazem in anticipation of further improvement. Her clinical symptoms continued to improve (WHO-FC I) concomitantly with normalization of BNP, and almost normalized peak $\mathrm{VO}_{2}$. The latest hemodynamic measurement (September 10 $0^{\text {th }}$ ) showed further improvement in PVR (328 dyne $\cdot \mathrm{sec} \cdot \mathrm{cm}^{-5}$ ) and almost normalized $\mathrm{mPAP}(18 \mathrm{mmHg})$ with preserved CI $\left(2.1 \mathrm{~L} \cdot \mathrm{min}^{-1} \cdot \mathrm{m}^{-2}\right)$. The abnormal electrocardiography findings disappeared, and the morphological deformities returned to approximately normal shape and proportion (Figures 2 and 3, respectively). The oral combination therapy was well tolerated and its therapeutic effectiveness was still present 9 months from the initial diagnosis.

\section{Discussion}

To our knowledge, there has never been a report about such a responder or a reported case that was improved with oral drugs only. Furthermore, the pulmonary vasoreactivity to inhaled NO recovered to almost a positive level. A positive level in an acute pulmonary vasoreactivity test generally indicates the significant contribution of vasoconstriction to the pathophysiology of $\mathrm{PAH}$, also implying less fixed structural obstruction in the pulmonary artery, and indicating the possible effectiveness of calcium channel blockers in IPAH patients. ${ }^{4}$ The clinical significance of a positive change in an acute vasoreactivity test after medical therapy has not been clearly defined, but may theoretically suggest an improvement in vasodilating ability. This restoration of the vasodilating activity may on the contrary imply that the vascular organic remodeling had not progressed markedly, which may be reasonable since her PAH was rapidly established. Another possibility is that the vaso-spasm was too strong to be relieved by nitric oxide (20 ppm), before the oral combination therapy. Combination use of drugs acting on various cascades may have strongly inhibited the constriction of the pulmonary arteries and the proliferation of endothelial and/or smooth muscle cells, although we did not have any direct proof, such as pathological assessment. Judging from the newest guidelines, ${ }^{2,3)}$ IV epoprostenol should have been recommended as a first-line therapy for such a severe patient, and the oral medications should have been additive ones for the sequential combination therapy if the effect of IV epoprostenol was not sufficient. ${ }^{3,5)}$ However, we selected the oral combination therapy as the first-line therapy for the following 3 reasons. Firstly, diuretics and oxygen supply had already showed some effects on her symptoms on admission to our hospital, providing time for attempting the oral drugs, although intravenous catecholamines were always ready for use in case of clinical worsening. Secondly, she was strongly opposed to IV epoprostenol administration without first trying the oral drugs. Thirdly, she consented to our request for careful and frequent hemodynamic evaluations and immediate introduction of IV epoprostenol if her clinical parameters worsened. Oral combination therapy has tremendous advantages for patients, which include the avoidance of catheter-induced infection, troubles with preparing the epoprostenol-solution by oneself, and the burden of carrying around accessories related to the pump. One clinical study suggested the combined use of bosentan, sildenafil, and inhaled iloprost in patients with severe PAH in conjunction with a goal-oriented strategy improved survival and decreased the combined endpoint of death, lung transplantation, and need for intravenous prostacyclin treatment. ${ }^{6}$ However, it is not clear at all whether the result means that the effect of the combination therapy was sufficient to achieve the normalization of pulmonary vasculature (reverse remodeling). Even though high-dose IV epoprostenol strongly inhibits the vasoconstriction and cell-proliferation of pulmonary vessels, it appears to be difficult to prevent the progression of pulmonary vascular remodeling without adequate reduction of pulmonary arterial pressure. ${ }^{7}$ It has been proposed that maintaining PAP and PVR at low levels may be essential for reverse remodeling, ${ }^{8,9)}$ but on the other hand, it seems almost impossible to restore normal hemodynamics with monotherapy using one of oral prostacyclin analogues, ERA, and PDE-5 inhibitors in IPAH patients. ${ }^{10)}$ Thus, initial and aggressive combination therapy whose aim is to obtain normal hemodynamics should be attempted and may achieve reverse remodeling. Indeed, we could achieve a substantial reduction in mPAP and PVR with large increases in CI in our IPAH patient with oral combination therapy, as well as normalization of the electrocardiogram and morphological deformities, suggesting reverse remodeling in the pulmonary vasculature and myocardium. We should not overlook any patient responding well to oral combination therapy before introducing IV epoprostenol therapy, although careful and frequent evaluations with invasive hemodynamic measurements may be required to ensure the correct timing of IV epoprostenol introduction.

\section{REFERENCES}

1. Galiè N, Manes A, Negro L, Palazzini M, Bacchi-Reggiani ML, Branzi A. A meta-analysis of randomized controlled trials in pulmonary arterial hypertension. Eur Heart J 2009; 30: 394-403. (Review)

2. Barst RJ, Gibbs JS, Ghofrani HA, et al. Updated evidence-based treatment algorithm in pulmonary arterial hypertension. J Am Coll Cardiol 2009; 54: S78-84. (Review)

3. Galiè N, Hoeper MM, Humbert M, et al. Guidelines for the diagnosis and treatment of pulmonary hypertension: the Task Force for the Diagnosis and Treatment of Pulmonary Hypertension of the European Society of Cardiology (ESC) and the European Respiratory Society (ERS), endorsed by the International Society of Heart and Lung Transplantation (ISHLT). Eur Heart J 2009; 30: 2493537.

4. Sitbon O, Humbert M, Jaïs X, et al. Long-term response to calcium channel blockers in idiopathic pulmonary arterial hypertension. Circulation 2005; 111: 3105-11.

5. McLaughlin VV, Archer SL, Badesch DB, et al. ACCF/AHA 2009 expert consensus document on pulmonary hypertension a report of the American College of Cardiology Foundation Task Force on Expert Consensus Documents and the American Heart Association developed in collaboration with the American College of Chest Physicians; American Thoracic Society, Inc.; and the Pulmonary Hypertension Association. J Am Coll Cardiol 2009; 53: 1573-619.

6. Hoeper MM, Markevych I, Spiekerkoetter E, Welte T, Niedermeyer J. Goal-oriented treatment and combination therapy for pulmonary arterial hypertension. Eur Respir J 2005; 26: 858-63.

7. Rich S, Pogoriler J, Husain AN, Toth PT, Gomberg-Maitland M, Archer SL. Long-term effects of epoprostenol on the pulmonary vasculature in idiopathic pulmonary arterial hypertension. Chest 2010; 138: 1234-9. 
8. Deb S, Yun J, Burton N, Omron E, Thurber J, Nathan SD. Reversal of idiopathic pulmonary arterial hypertension and allograft pneumonectomy after single lung transplantation. Chest 2006; 130: 214-7.

9. Levy NT, Liapis H, Eisenberg PR, Botney MD, Trulock EP. Path- ologic regression of primary pulmonary hypertension in left native lung following right single-lung transplantation. J Heart Lung Transplant 2001; 20: 381-4.

10. Rich S. The current treatment of pulmonary arterial hypertension: time to redefine success. Chest 2006; 130: 1198-202. (Review) 